\title{
Criterios básicos \\ para la evaluación del aprendizaje de la Geografía
}

\author{
antonio luis Garcia Ruiz *
}

\section{INTRODUCCION}

En nuestro actual sistema de enseñanza universitaria, los exámenes siguen siendo una de las claves más importantes; éstos constituyen el tema principal de las discusiones entre profesores y alumnos que, en la mayoria de los casos, casi nunca se ponen de acuerdo en la forma de realizarlos. Aceptado ya, mayoritariamente, lo antipedagógico que resulta calificar a los alumnos a través de un solo examen a final de curso, o incluso tres, uno al finalizar cada trimestre, aun no se ha encontrado una forma clara y coherente de evaluar, que sea aceptada por la mayoria de docentes y discentes. La verdad es que en la Universidad, aún no se ha asumido plenamente lo que significa la evaluación en su verdadero sentido, aunque quizá sea la Geografía una de las materias que más intentos de renovación didáctica ofrece.

Con frecuencia comprobamos que a nuestros alumnos les importa más la nota que el aprendizaje de la materia correspondiente; una de las preguntas que anualmente se repiten al comienzo del curso, es la de cómo van a ser los exámenes. Sus intereses giran en torno a ellos; valoran a cada profesor más por su forma de evaluar, que por la de enseñar.

Evidentemente, no podemos estar de acuerdo con estos planteamientos, por lo que compartiendo la idea de otros profesores, pensamos que cen una sociedad en la que el valor social y personal de las calificaciones es tan

(*) Escuela Universitaria de Formación del Profesorado. Granada. 
extraordinario, parece lógico y hasta de justicia prestar un interés paralelo por parte del profesor a las técnicas y procedimientos de evaluación" (S. MOLINA: Didáctica General, Madrid 1983).

En esta comunicación pretendemos dar a conocer nuestra experiencia evaluadora, con el fin de que sirva para dilucidar y encontrar la mejor forma de evaluar el aprendizaje de la Geografía de las Facultades y Escuelas Universitarias, donde los horarios son muy limitados y el número de alumnos muy alto.

Esta experiencia la hemos realizado con los alumnos de segundo y tercer curso de Magisterio de la Escuela Universitaria de Formación del Profesorado de Granada.

\section{FUNCION DE LA EVALUACION}

La evaluación es esencialmente una comprobación de objetivos conseguidos. Nos sirve también para llevar un control del proceso de aprendizaje y de nuestro propio rendimiento como profesores. A. A. FRANQUEIRO la define como «la etapa del proceso de aprendizaje que permite determinar en qué medida se han logrado los objetivos propuestos" (La enseñanza de las Ciencias Sociales, Buenos Aires 1984).

Pero la evaluación no es un apéndice del proceso de aprendizaje, sino que está integrada en él; su función no es únicamente comprobar rendimientos, sino también valorar y reorientar de forma continua el sistema de enseñanza, en busca siempre de una mayor eficacia y calidad. Sin embargo, de nada sirve la evalución si antes no hemos programado correctamente todos los demás elementos integrantes del proceso de aprendizaje, fundamentalmente los objetivos. A. GONZALEZ (Didáctica de las Ciencias Sociales, Barcelona 1980) nos indica en este sentido: "Queremos dejar bien claro que de nada sirven las mejoras evaluativas ni la técnica docimológica, aunque necesaria, si el mal es de origen: falta de fines, incongruencia de objetivos, desestructuración del contenido".

La evaluación nos debe servir de instrumento crítico permanente al sistema de enseñanza, para introducir todas las mejoras y cambios que el mismo requiera; como dice B. ROTGER: "La evaluación es, por tanto, una 
actividad sistemática y permanente, integrada en el proceso educativo, con el fin de mejorar este proceso, ayudar y orientar al alumno, criticar y revisar los planes y programas, los métodos y los recursos" (El proceso programador, Madrid 1979).

\section{LOS CUATRO CRITERIOS BASICOS UTILIZADOS}

Además de cumplir todas las funciones que acabamos de señalar, entendemos que la evaluación ha de ser completa, es decir, que abarque todos los aspectos de la materia que estamos estudiando; en nuestro caso cualquier asignatura de la rama de Geografía. Con este fin hemos empleado cuatro criterios de evaluación para responder a los cuatro aspectos fundamentales que en las diversas asignaturas geográficas hemos de enseñar.

Primero. Un conjunto de conocimientos que los alumnos han de adquirir en función del programa impartido, y que incidirán, sobre todo, en los conceptos, ideas y teorías fundamentales, así como en una terminología básica y correcta, y en unos datos mínimos e imprescindibles. Para comprobar el aprendizaje de este contenido teórico hemos utilizado el examen escrito.

Segundo. Dominio de una serie de métodos, destrezas y técnicas de trabajo en Geografia: representaciones cartográficas y en relieve, husos horarios y de escalas, realización e interpretación de gráficos y diagramas climatológicos, demográficos, económicos, etc., cálculo de índices, aplicación de fórmulas, manejo de aparatos, elaboración de cuadros, catalogación de materiales, análisis y comentario de diapositivas y fotografias aéreas, etc. Para evaluar el dominio de estos ejercicios empleamos el examen de prácticas.

Tercero. Ampliación y profundización en los conocimientos a fin de elevar el nivel cognoscitivo, intelectual y cultural del alumno. Este aspecto lo fomentamos y lo comprobamos a través de la lectura de libros.

Cuarto. Iniciación a la investigación en Geografía, que se consigue y valora con la realización de trabajos en equipo. 
Estos son, pues, los cuatro criterios fundamentales que entendemos deben emplearse en la evaluación de la enseñanza geográfica. Cada uno de ellos debe calificarse de cero a diez puntos, como es habitual, y la nota final debe ser la media de estas cuatro. Así todos los aspectos se valoran en la misma proporción, y sólo hacemos exigencia de un requisito: que en cada uno de los cuatro aspectos, habrá de obtener el alumno una calificación mínima de cinco.

Vistos cuáles son los criterios empleados para evaluar, examinemos por separado cómo utilizamos cada uno de ellos.

\section{EXAMEN DE TEORIA}

El examen de teoría, que generalmente se hace escrito, aunque también puede hacerse oral, nos sirve para evaluar los conocimientos adquiridos por los alumnos, que han sido expuestos y debatidos en clase anteriormente y facilitados en fotocopia a los mismos, asi como para comprobar su capacidad de expresión, su forma de presentar y organizar ideas, propias o ajenas, la manera de razonar, la de conectar hechos y fenómenos, etc.

Aunque pueda parecer que nos encontramos ante un examen de corte tradicional, no es asi ni muchos menos, y por varias razones:

- La materia se subdivide en partes unitarias para hacer un examen de cada una de ellas, por lo que no es tan extensa como en los mencionados exámenes tradicionales.

- En el examen no se pide una reproducción más o menos precisa de los apuntes, sino unas respuestas sazonadas y un tanto personales, sobre los conceptos básicos e ideas fundamentales que se pretenden conocer, aunque a veces haya de incluirse un mínimo imprescindible de información.

- El tiempo para responder es ilimitado.

- Se le dan dos oportunidades para recuperar la materia suspendida. 
- La nota media final de todos los exámenes constituye la cuarta parte de la nota de fin de curso.

Así pues, con estas características, los alumnos realizan gustosos los exámenes y responden positivamente en un índice muy elevado de los mismos: el 78,5 por 100 aprueban en el primer examen, el 16,5 por 100 lo hacen en la primera recuperación, el 0,5 por 100 en la segunda recuperación y el 4,5 por 100 restante no se examinan en alguno de los tres exámenes.

\section{EXAMEN PRACTICO}

Las clases prácticas se van alternando con las teóricas y van en consonancia y en función de estas segundas. De cada ejercicio práctico o grupo de prácticas, se realiza un examen, que es simplemente una práctica más, sólo que corregida y calificada por el profesor. Cada uno de los exámenes, al igual que en la teoría, son eliminatorios y es necesario aprobarlos todos para que la nota final de prácticas sea de aprobado.

Las prácticas pueden impartirse por el mismo profesor de teoría - como en nuestro caso- o por otro profesor del Departamento que trabaje de acuerdo con él.

En el examen de prácticas puede y debe utilizarse todo tipo de materiales e instrumentos útiles para el aprendizaje y la investigación en Geografía.

\section{EXPOSICION DE LOS LIBROS LEIDOS}

La lectura de libros constituye la mejor forma de profundizar en los temas, completar sus contenidos y poner al día los conocimientos. Al mismo tiempo habitúan a nuestros alumnos a la lectura; a pesar de ser universitarios, la mayor parte de ellos la practican con muy poca frecuencia. 
Hemos exigido la lectura de una obra en cada trimestre y por cada asignatura.

A comienzos del curso se les facilita una bibliografía general de la materia. Además, en los apuntes, cada tema lleva su bibliografía específica. Así, los alumnos poseen una bibliografia suficiente como para poder elegir los títulos que deseen; si la asignatura se compone de partes diferentes, es necesario indicar que cada uno de los tres libros que han de leerse pertenezcan a partes diferentes de la materia, para que no se lean varios libros de una y ninguno de otra.

La evaluación de la lectura se realiza mediante la exposición y comentario del resumen de cada obra, que el alumno hace individual y verbalmente al profesor, fuera de las horas de clase. Este sistema nos ocupa mucho tiempo pero proporciona resultados altamente positivos, pues ya no sólo se califica la lectura, también se intercambian ideas y opiniones con el alumno, lo que resulta tremendamente enriquecedor $y$, sobre todo, orientador, pues nos ayuda a conocerlos y saber sus deseos y preferencias.

\section{TRABAJOS DE INICIACION A LA INVESTIGACION}

Este tipo de trabajos son hoy muy frecuentes en la enseñanza de la Geografia. Presentan unas ventajas que los hacen convenientes, y también necesarios:

- Los alumnos se inician en el método de investigación científica, que en el futuro pueden desarrollar.

- Fomentan el trabajo en equipo, única forma de investigar y trabajar con el rigor que la ciencia requiere.

- Favorece el trabajo interdisciplinar, pues a pesar de que todos cursan la misma especialidad, cada uno estudia más un aspecto determinado, como si de un equipo de diversos especialistas se tratase. 
En cualquier caso, siempre habrá que darles, previo al comienzo de los trabajos, un esquema con los principales pasos y fases del método de investigación científica para que lo apliquen en la medida que les sea posible.

Es preferible que los temas de trabajo sean elegidos por ellos mismos, aunque también habrá que orientarles sobre ello. Evidentemente han de ser temas inéditos. Al mismo tiempo su investigación no debe presentar gran dificultad, puesto que si es así "se pierden" y no llegan a hacer nada. Una vez terminados deben ser expuestos en clase y corregidos y calificados por el profesor.

\section{CONCLUSIONES}

Esta forma de evaluar el aprendizaje de la Geografía ha demostrado poseer una serie de ventajas que nos hacen considerarla, por el momento, como la más adecuada para nuestros alumnos:

- Con ella queda desterrado, en su faceta más negativa, el abuso que se ha venido haciendo de los exámenes memorísticos y que producian escaso aprendizaje en los alumnos.

- Al mismo tiempo se realiza un estudio completo de la materia y una evaluación igualmente completa, y más coherente.

- También se alcanza una forma de evaluar más continua, más atractiva y más fácil para los alumnos, pero al mismo tiempo, más instructiva.

Finalmente, como conclusión más importante diremos que se obtienen unos resultados altamente satisfactorios: el 17,6 por 110 de los alumnos obtienen la calificación de sobresaliente, el 41,3 por 100 la de notable, el 35,7 por 100 la de aprobado, y el 5,4 por 100 resultan suspensos.

Estas notas no sólo son más elevadas, sino, lo que es más importante, reflejan más fielmente el grado de aprendizaje adquirido por los alumnos. 


\section{BIBLIOGRAFIA}

BAILEY, P: Didáctica de la Geografia, Cincel-Kapelusz, Madrid 1981.

Benito, V. y Otro: Evaluación aplicada a la enseñanza, C.E.A.C. Barcelona 1977.

Franqueiro, A.A.: La enseñanza de las Ciencias Sociales, El Ateneo, Buenos Aires 1984.

González Hernández, A.: Didáctica de las Ciencias Sociales, C.E.A.C. Barcelona 1980.

Herrero Fabregat, Cl.: Cómo preparar las clases de Geografía, Anaya, Madrid 1980.

I.E.P.S.: Las Ciencias Sociales, Narcea, Madrid 1976.

LINDAWALL y otros: Cómo evaluar el curriculum, Ateneo, Buenos Aires 1974.

LoGAN, L.M.: Estrategias para una enseñanza creativa, Oikos-Tau, Barcelona 1980.

LLOPIS, Carmen: Las Ciencias Sociales en el aula, Narcea, Madrid 1982. 
Criterios básicos para la evaluación del aprendizaje de la Geografía

Pérez Picazo, M. ${ }^{a}$ T.: Didáctica de las Ciencias Sociales, H.S.R., Burgos 1975.

Rotger, B.: El proceso programador en la escuela, Escuela Española, 1979.

SAEZ, O. y otros: Didáctica General, Anaya, Madrid 1983. 